\title{
A study of incidental adnexal masses during caesarean section and its management
}

\author{
Ashok Kumar K, Harshini V, Sreelatha S, Ramya S, Rajeshwari \\ Correspondence: Dr Sreelatha S, Associate Professor, Department of Obstetrics and \\ Gynaecology, ESIC MC PGIMSR, Rajaji Nagar, Bangalore, India; Email - \\ dr.sreelatha2011@gmail.com
}

Distributed under Creative Commons Attribution-Share Alike 4.0 International.

\begin{abstract}
Objectives: The aims of the study were to observe the incidental adnexal masses and its management during caesarian section. Methods: A retrospective study conducted in Department of Obstetrics and Gynecology, ESIMC PGI MSR Bangalore, Karnataka. Data collected from forty pregnant women during caesarean section and incidentally found adnexal mass and its management. Result: Out of 40 women, 15 were primigravida, 25 were multigravida. Study results shows presence of clear cyst in 11 women, fimbrial cysts in 11 women, mucinous cystadenoma in 6 , serouscyst adenoma in 3 , corpus luteal cyst in 5 , hemorrhagic cyst in 2 , dermoid in 1, paraovarian cyst in 1, hydatid cyst of morgagni in 1. Conservative approach was followed for 26 women, while cystectomy was performed in 10 women; fluid aspiration was done in 4 women. Conclusion: Adnexal masses, which arise in pregnancy, are mostly functional and asymptomatic and resolve spontaneously. Surgical management of adnexal masses during caesarean section should be considered in some cases to avoid later surgery for this incidental pathology.
\end{abstract}

Keywords: Caesarian section, adnexal mass.

Adnexal masses originate in the ovaries, fallopian tubes, or surrounding tissue. Adnexal masses can be found in women of any age, and pregnancy is no exception. The reported incidence of adnexal masses during pregnancy varies from 1 in 81 pregnancies to 1 in 8000 pregnancies ${ }^{1}$. With routine obstetric ultrasound examinations, ovarian cysts are now more commonly diagnosed during pregnancy and their management is still a challenging clinical issue among obstetricians ${ }^{2}$. Although most of the adnexal masses are pregnancy related and may resolve by the $16^{\text {th }}$ week of gestation, the management of the masses that persist is still controversial $^{3}$. They may present as different histological types and their clinical significance may vary largely depending mainly on tumor size, clinical presentation, and risk of malignancy.

Routine ultrasound scans can reveal adnexal masses in early pregnancy up to $1-2 \%$ of total pregnancies 4 . The management of this rare condition during pregnancy is important. Patients usually present with abdominal pain in lower quadrants. It is hard to make a decision about operation in pregnancy because of postoperative early fetal loss. There are three reasons for surgery of adnexal masses during pregnancy; (i) eliminating of a possible distocia, (ii) danger of torsion, rupture or haemorrhage, (iii) risk of malignancy ${ }^{5}$. Earlier studies recommend

Received: 22 nd September 2017. Accepted: 7 th December 2017.

Ashok Kumar K, Harshini V, Sreelatha S, Ramya S, Rajeshwari. A study of incidental adnexal masses during caesarean section and its management. The New Indian Journal of OBGYN. 2018; 4(2): $161-64$. 
excision of adnexal mass at 16-18 weeks gestation to avoid the risk of abortion that frequently occurs if surgery is performed in the first trimester ${ }^{6}$. Currently, advances in ultrasound technology enable us to detect more often adnexal masses during pregnancy. In contrast to early gestation, in the third trimester a gravid uterus may obscure the correct visualization and detection of an adnexal mass or focusing on the baby and placenta may keep us from the detection of adnexal pathology and moreover, there may be technical difficulties in evaluating velocimetric features during pregnancy, as the vessels and blood flow surrounding the gravid uterus mainly have high velocity and low resistance characteristics.

Usually adnexal masses in pregnancy are imagiological findings, but when detected by palpation on physical exam, ultrasound should be the first step to characterize the lesion. Transabdominal and/or transvaginal ultrasound allows morphodimensional evaluation and assessment of mass vascularization and growth, as well as observation of the contralateral ovary and detection of other malignant signs, such as the presence of ascites and peritoneal carcinomatosis ${ }^{7,8}$. The interpretation of tumor markers serum levels in pregnant women requires caution, since they may be physiologically increased during certain stages of pregnancy. This is particularly true regarding some oncofetal antigens (alpha-fetoprotein, human chorionic gonadotropin, and CA125), which are involved in biological functions associated with development, differentiation, and fetal maturation ${ }^{9-12}$. The main interest of tumor markers seems to be the differentiation between primary and secondary adnexal tumors and the possibility to monitor their levels to evaluate the therapeutic response and the risk of relapse ${ }^{13}$.

\section{Method}

A retrospective study conducted in Department of Obstetrics and Gynecology, ESIMC PGI MSR Bangalore, Karnataka. Data collected from forty pregnant women during caesarean section and incidentally found adnexal mass and its management. All clinical, intraoperative and pathological findings are taken from hospital records retrospectively.

\section{Results}

Out of 40 women, 15 were primigravida, 25 were multigravida. Study results shows presence of clear cyst
Table 1: Type of cyst

\begin{tabular}{ll}
\hline Histopathology & Number of women \\
\hline Clear cyst & 10 \\
Fimbrial cyst & 11 \\
Corpus luteal cyst & 5 \\
Hemorrhagic cyst & 2 \\
Hydatid cyst of morgagni & 1 \\
Dermoid & 1 \\
Mucinous cyst & 6 \\
Serous cystadenoma & 3 \\
Paraovarian cyst & 1 \\
\hline
\end{tabular}

in 11 women, fimbrial cysts in 11 women, mucinous cystadenoma in 6 , serous cystadenoma in 3 , corpus luteal cyst in 5 , hemorrhagic cyst in 2 , dermoid in 1 , paraovarian cyst in 1 , hydatid cyst of morgagni in 1 (Table 1 ).

Table 2: Management

\begin{tabular}{ll}
\hline Management & Number of women \\
\hline Cystectomy & 10 \\
Puncture/aspiration & 4 \\
Conservative & 26 \\
Total & 40 \\
\hline
\end{tabular}

Conservative approach was followed for 26 women, while cystectomy was performed in 10 women; fluid aspiration was done in 4 women (Table 2). No carcinoma was found.

\section{Discussion}

Adnexal masses can be diagnosed in pregnancy (up to $1-2 \%$ of all pregnancies) with the help of ultrasound examination. As many of these cysts will spontaneously resolve by the second trimester and the risk of malignancy is low, a conservative management during pregnancy is advised. Ultrasound has a great value in the diagnosis and monitoring of adnexal masses in the first and beginning of the second trimesters of pregnancy, but later on, as the uterus enlarges, the correct diagnosis is hard to establish ${ }^{14}$. Obstetricians can find themselves facing another dilemma during cesarean section when they incidentally discover an adnexal mass regarding the medico-legal issues on the informed consenting process ${ }^{15}$.

Most of the patients didn't have antenatal diagnosis of the adnexal mass, making the finding at caesarean section time an incidental one. Literature indicates dermoid cyst to be the most common pathological finding, but the percentage varies considerably $(28-50 \%)^{16}$. 
Dede and Hoffman conclude that at the time of cesarean section incidental adnexal masses should be surgically removed, avoiding later surgery and establishing malignancy status of the mass. Leiserowitz adds that ovarian masses should be removed intact when possible, especially in suspicious cases. Cystectomy is the recommended procedure, ovariectomy and ovariectomy with salpingectomy being reservated for complex cases.

In case of suspicion of malignant masses during the surgical examination, Surgical treatment of the adnexal masses has to be performed with adequate staging and debulking equal to the treatment of non-pregnant women. However, whereas during organogenesis abortion has to be considered prior to chemotherapy, later in pregnancy surgical debulking as complete as possible, followed by taxol-platinum chemotherapy is indicated. If the fetus is not viable at the time of primary surgery, neoadjuvant chemotherapy and complementation of surgery after delivery of the baby should be performed. It should be stressed that chemotherapy for ovarian cancer applied during pregnancy appears to be safe. However, no studies have evaluated the long-term consequences for children exposed to intra-uterine chemotherapy. Aspiration of cysts should be avoided, as the correlation between the histological evaluation of an ovarian malignancy and the cytological evaluation of aspirates is poor. Moreover, spillage of malignant cysts is hazardous for the patient ${ }^{17}$.

\section{Conclusion}

Adnexal masses, which arise in pregnancy, are functional and asymptomatic and resolve spontaneously. Persistent adnexal masses carry a low risk of malignancy, and ultrasonography is the preferred method to assess this risk. On the other hand when strong clinical suspicion of malignancy or presence of acute abdomen or severe clinical manifestations occurs, surgical treatment is indicated by laparotomy or laparoscopy. Surgical management of adnexal masses during caesarean section should be performed to avoid later surgery for this incidental pathology.

\section{Conflict of interest: None. Disclaimer: Nil.}

\section{References}

1.Grimes WH Jr, Bartholomew RA, Colvin ED, Fish JS, Lester WM. Ovarian cyst complicating pregnancy. Am J Obstet Gynecol. 1954; 68: 594-605.

2.El-Shawarby SA, Henderson AF, Mossa MA. Ovarian cysts during pregnancy: Dilemmas in diagnosis and management. J Obstet Gynaecol. 2005; 25(7): 669-75.

3.Dursun P, Yanık FF, Cabuk E, Zeyneloglu HB, Bildaci B, Kuscu E, et al. Adnexal masses in pregnancy: Baskent University experience. J Turk Soc Obstet Gynecol. 2011; 8: 113-7.

4. White KC. Ovarian tumors in pregnancy. A private hospital ten year survey. Am J Obstet Gynecol. 1973; 116: 544-50.

5.Mundell EW. Primary ovarian cancer associated with pregnancy. Clin Obstet Gynecol. 1963; 6: 983-93.

6. White KC. Ovarian tumors in pregnancy. A private hospital ten year survey. Am J Obstet Gynecol. 1973; 116: 544-50.

7.Zanetta G, Mariani E, Lissoni A, et al. A prospective study of the role of ultrasound in the management of adnexal masses in pregnancy. BJOG: An International Journal of Obstetrics and Gynaecology. 2003; 110(6): 578-583. doi: 10.1046/j.1471-0528.2003.02940.x.

8.Glanc P, Salem S, Farine D. Adnexal masses in the pregnant patient a diagnostic and management challenge. Ultrasound Quarterly. 2008; 24(4): 225-40.

9.Sarandakou A, Protonotariou E, Rizos D. Tumor markers in biological fluids associated with pregnancy. Critical Reviews in Clinical Laboratory Sciences. 2007; 44(2):15178.

10.Aslam N, Ong C, Woelfer B, Nicolaides K, Jurkovic D. Serum CA125 at 11-14 weeks of gestation in women with morphologically normal ovaries. BJOG: An International Journal of Obstetrics \& Gynaecology. 2000; 107(5): 689-90. doi: 10.1111/j.1471-0528.2000.tb13314.x.

11.Sarandakou A, Kontoravdis A, Kontogeorgi Z, Rizos D, Phocas I. Expression of CEA, CA-125 and SCC antigen by biological fluids associated with pregnancy. European Journal of Obstetrics and Gynecology and Reproductive Biology. 1992; 44(3): 215-20.

12.Sarandakou A, Phocas I, Botsis D, Rizos D, Trakakis E, Chryssikopoulos A. Vaginal fluid and serum CEA, CA125 and SCC in normal conditions and in benign and malignant diseases of the genital tract. Acta Oncologica. 1997; 36(7): 755-59. doi: 10.3109/02841869709001350. 
The New Indian Journal of OBGYN. 2018 (January-June); 4(2)

13.Hoover K, Jenkins TR. Evaluation and management of adnexal mass in pregnancy. American Journal of Obstetrics and Gynecology. 2011; 205(2): 97-102. doi: 10.1016/j.ajog.2011.01.050.

14.Nick AM, Schmeler K. Adnexal Masses in Pregnancy. Perinatology. 2012; 1(2):13-21

15.Horowitz NS. Management of adnexal masses in pregnancy. Clin Obstet Gynecol. 2011; 54(4): 519-27.

16.Paredes I, Pastrana M, Gordon A, Lick Tan T. Incidental adnexal mass at caesarean section- the value of implementing a comprehensive consenting process. BJMP. 2011; 4(1): a409
17.Hermans RHM, Fischer DC, van der Putten HWHM, van de Putte G, Einzmann T, et al. Adnexal Masses in Pregnancy. Onkologie. 2003; 26(2):167-72.

Ashok Kumar $\mathrm{K}^{1}$, Harshini $\mathrm{V}^{2}$, Sreelatha $\mathrm{S}^{3}$, Ramya $S^{4}$, Rajeshwari ${ }^{5}$

${ }^{1}$ Associate Professor, ${ }^{2}$ Senior Resident, ${ }^{3}$ Associate Professor, ${ }^{4}$ Junior Resident, ${ }^{5}$ Junior Resident; Department of Obstetrics and Gynaecology, ESIC MC PGIMSR, Rajaji Nagar, Bangalore, India 